\title{
AUTOMATIC CONTROL STRATEGIES IMPLEMENTED ON A WATER CANAL PROTOTYPE
}

\author{
João Figueiredo*; M. Ayala Botto ** \\ *Universidade de Évora,IDMEC, jfig@uevora.pt; \\ **Technical University of Lisbon, Instituto Superior Técnico,IDMEC, migbotto@dem.ist.utl.pt
}

\begin{abstract}
Water canals are complex hydraulic systems because they are big scale systems, open and characterized by big delays and great inertia. Many models and control strategies have already been simulated using linear control theory. In the present study, a PI controller is experimentally implemented in a modern automated canal prototype where sensors and actuators are controlled via a PLC network supervised by a SCADA system. This study shows the great potential of modern control technology applied to water distribution systems as the water resource is becoming a major valuable asset in today societies. This paper will serve as a basis knowledge to fully use this modern canal capabilities as it provides this automatic facility with the basic tools where more sophisticated control strategies can be, stepwise added, in the future. Copyright $@ 2005$ IFAC
\end{abstract}

Keywords: Modelling, Control, Automatic Irrigation Canals, PLC and SCADA Programming

\section{INTRODUCTION}

It is nowadays clear and well accepted that water resources are becoming scarce and, in the near future, will actually present one of the biggest problems that modern societies have to face.

Irrigation water is the main use of water resources and so, better conveyance efficiencies as well as intelligent management of open-channel conveyance and delivery systems are main goals to achieve within a short period of time.

Upstream control canals are only efficient when operates with rigid water delivery methods (Rijo, 1999). Nevertheless, a great part of these systems work with flexible water delivery schedules and, in that case, operational losses become much more significant (Rijo, 1999).

One of the several ways to try to achieve a better efficiency in irrigation canals is to provide automatic systems of control and monitoring to this kind of facilities. This paper presents a standard control strategy based on PI controllers that was experimentally tested to control a modern automatic irrigation canal prototype.
These control modes are implemented through a PLC network installed on the automatic canal which is supervised by a SCADA software system (Supervisory Control And Data Acquisition).

\section{BRIEF DESCRIPTION OF THE FACILITY}

\subsection{Automatic Canal}

The experimental automatic canal is located in the University of Évora, Portugal (Fig.1).

(http://canais.nuhcc.uevora.pt).

The canal has four pools, measuring roughly $40 \mathrm{~m}$ each, and the geometry of its cross section is trapezoidal (Fig. 2). The hydraulic features used in project, as well as in all the following analysis, were a flow of $0,090 \mathrm{~m}^{3} / \mathrm{s}$ for a uniform water depth of $0,700 \mathrm{~m}$. The water in this experimental canal flows in closed circuit, regarding water savings; the return to the storage reservoir is guaranteed by a traditional irrigation canal (fig 3).

The four pools of the automatic canal are divided by three sluice gates; the last one ends with an overshot gate, which discharges to the referred traditional 
canal. Immediately upstream of each gate there is an orifice type offtake, equipped with a flowmeter, and a counterweight-float level sensor (Figure 4); a servo-motorised valve controls the flow in the offtake. The gate is also motorised and both equipments have position sensors.

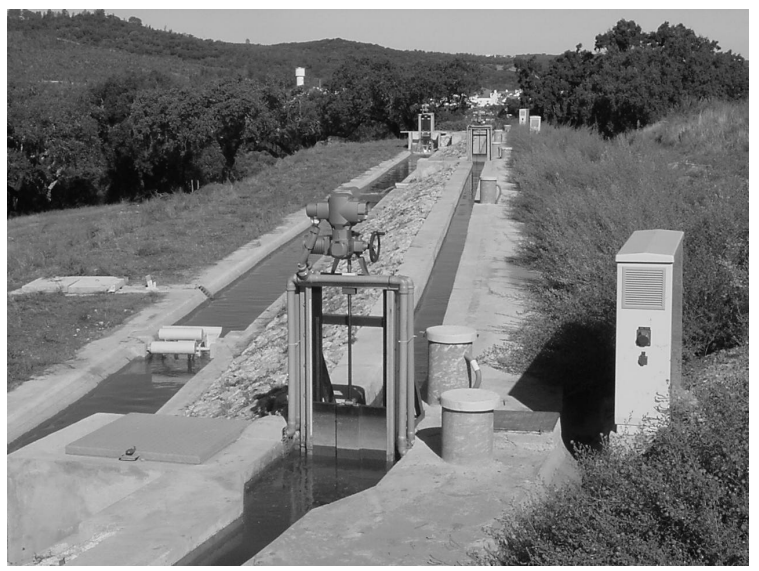

Fig. 1 - Automatic Canal Prototype

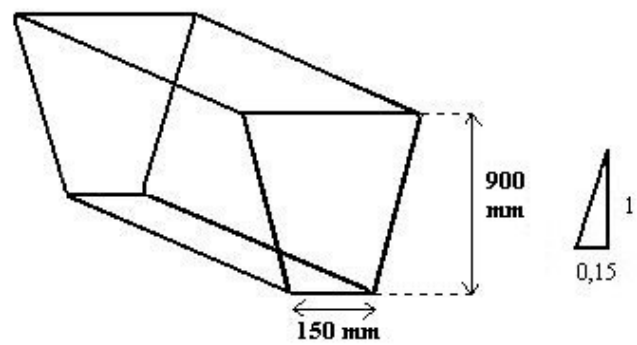

Fig. 2 - Geometry of a canal pool



Fig. 3 - Traditional irrigation canal

The flow within the automatic canal is regulated by another servo-motorised valve located at the exit of an high reservoir (head of the automatic canal), simulating a real load situation. This high reservoir is filled with the recovered water pumped from a low one, which collects the flow from the traditional canal. The schematic layout of the complete facility is shown in Fig. 5.

Regarding water savings, all offtakes discharge towards the traditional canal. Therefore, the installation has no losses of water except evaporation effects which are not, in this case, significant.

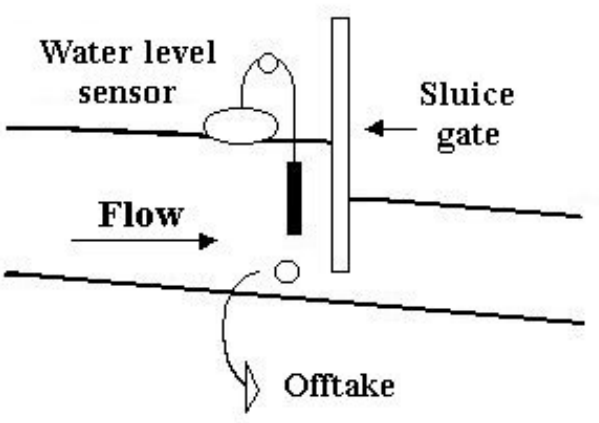

Fig. 4 - Schematics of the electromechanical equipment present in each gate off-take

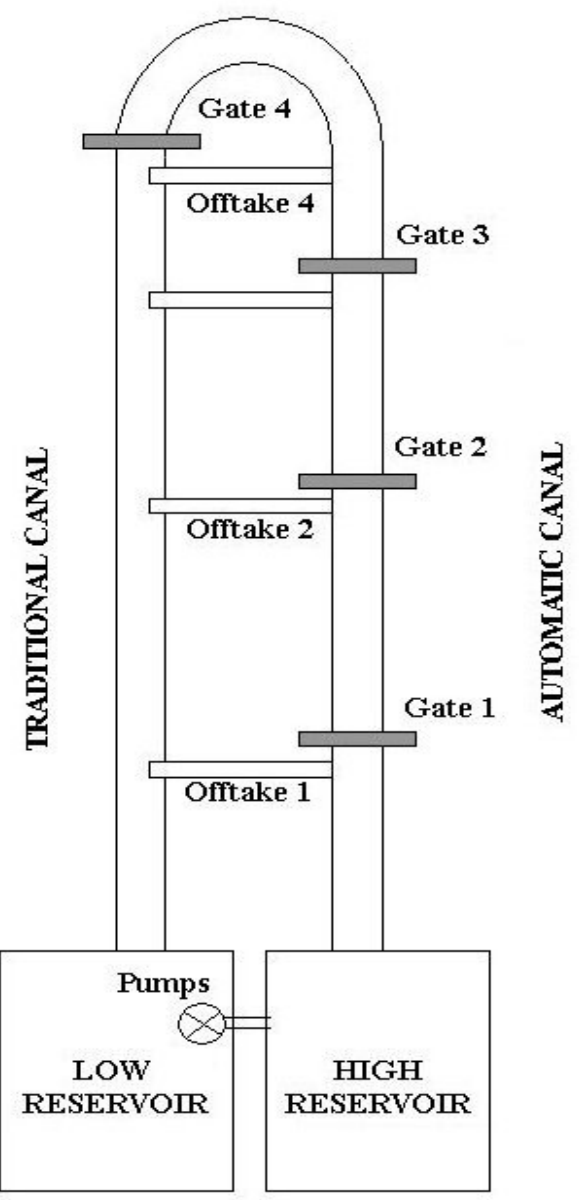

Fig. 5 - Schematics of the complete facility

Besides these hydraulic equipments, each group gateofftake is controlled and monitorised with PLCs responsible for all data acquisition and transfer (analogic and digital signals) as well as the control actions after appropriate programming (Fig. 6). The traditional canal has also a couple of cross-section regulators and two offtakes for demonstrating purposes. These equipments were not mentioned earlier because the following study will only involve automatic control chains. It is noticeable the versatility of this experimental facilities on the field of irrigation canal studies once one can choose the length of each reach, modifying its hydraulic behaviour and simulate several demand scenarios. 


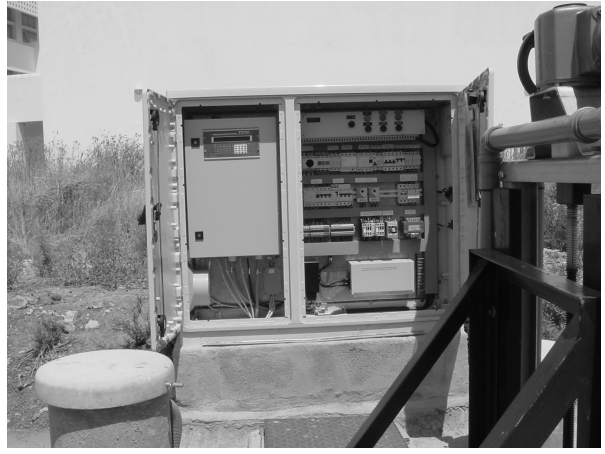

Fig. 6 - Hardware assigned to each gate/ off-take

\subsection{PLC Network and SCADA supervisor}

The installed PLC network (fig. 7) consists of 5 slaves PLCs ( 1 to 5: one for each gate off-take group and the fifth to control the pumps and main reservoir) connected to a master PLC via MODBUS network (RS 485).

Each slave PLC hosts several control programs which selection is made either locally, via an HMI (Human Machine Interface) or remotely, via the master PLC (PLC 0), which is connected to a server $\mathrm{PC}$, via RS232, where the SCADA application is running. The server $\mathrm{PC}$ is simultaneous a SCADA server and an internet server, as the implemented SCADA application is web enabled. All process variables are available at the SCADA PC as these variables are on-line available through a MODBUS connection protocol.

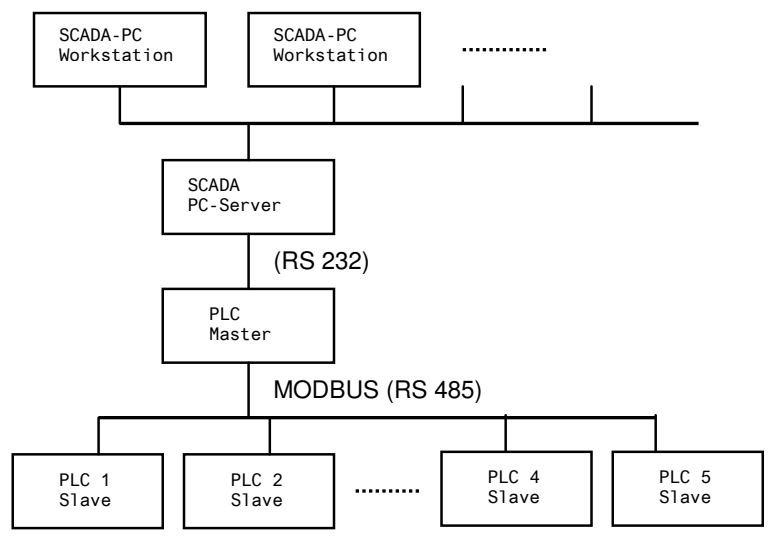

Fig. 7 - PLC Network and SCADA supervisor

\section{MATHEMATICAL MODEL}

\subsection{Basic Equations}

The dynamic behavior of hydraulic systems can be well described by a set of equations known as the Saint-Venant equations (Rijo, et al., 1992):

$$
\begin{aligned}
& \frac{\partial Q}{\partial x}+B \frac{\partial H}{\partial t}=0 \\
& \frac{\partial Q}{\partial t}+\frac{\partial}{\partial x}\left(\frac{Q^{2}}{A}\right)+g . A \cdot(J-i)=0
\end{aligned}
$$

where $Q$ is the discharge, $H$ the water depth, $B$ the water surface width, $A$ the water cross-section area, $g$ the gravity acceleration, $x$ the longitudinal abscissa in the direction of flow, $t$ the time, $i$ the bottom slope and $J$ the energy gradient slope that can be accurately approximated by the Manning-Strickler formula:

$$
J=\frac{Q^{2}}{K^{2} A^{2} R^{\frac{4}{3}}}
$$

where $K$ is the Manning-Strickler coefficient and $R$ the hydraulic radius defined by $R=A / P$, where $P$ is the wetted perimeter.

These equations are strongly non-linear and, for real canal systems, have no known analytical solution.

\subsection{Linearization}

Usually these equations are linearized assuming conditions near some steady state. This ensures that, for small deviations from a considered setpoint, the resulting equations will still describe the behaviour of the system.

The linearization of the Saint-Venant equations leads to (Schuurmans, 1997):

$$
\begin{aligned}
& \frac{\partial q}{\partial x}+B_{0} \frac{\partial h}{\partial t}=0 \\
& \frac{\partial q}{\partial t}+2 V_{0} \frac{\partial q}{\partial x}+\left(c_{0}^{2}-V_{0}^{2}\right) B_{0} \frac{\partial h}{\partial x}- \\
& \quad-\xi_{0} q-\gamma_{0} h=0
\end{aligned}
$$

where $q$ and $h$ are the variations of discharge and water depth, respectively, from a considered steady state, $c$ the wave celerity $(c=\sqrt{g A / B}), V$ flow velocity $(V=Q / A)$ and ${ }_{o}$ subscript stands for steady state values; $\xi_{0}$ and $\gamma_{0}$ are factors due to linearization defined by

$$
\begin{aligned}
& \xi_{0}=-\frac{2 g Q_{0}}{K^{2} A_{0} R_{0}^{\frac{4}{3}}}+\frac{2 Q_{0}}{A_{0}^{2}} B_{0}\left(\frac{d H}{d x}\right)_{0} \\
& \gamma_{0}=g i B_{0}\left[1+c o-\left(1+c o+(c o-2) F r^{2}\right) a\right] \\
& a=\frac{\left(\frac{d H}{d x}\right)_{0}}{I} \\
& c o=1+\frac{4}{3} \frac{P_{0}}{B_{0}}\left(\frac{d R}{d H}\right)_{0}
\end{aligned}
$$

where $F r$ is the Froude number defined by $F r=V / c$.

In spite of having now all the necessary equations linearized, there are still many steady state parameters left to determine, exception made to steady state discharge that one can choose freely as it is the condition for linearization.

Steady state parameters can be calculated by taking again the Saint-Venant equations and assuming no variations in discharge and flow depth in time. So, solving them leads to (Rijo, et al., 2002):

$$
\frac{d H}{d x}=\frac{J-i}{1-F r^{2}}
$$


This equation represents the spatial gradually varied flow and will be used to determine the flow profile in steady state flow conditions. Nevertheless, it is necessary to solve it by a numerical method, once it has no analytical solutions. As mentioned before, the values assumed are the project nominal features of the canal, i.e., discharge of $0,090 \mathrm{~m}^{3} / \mathrm{s}$ and $0,070 \mathrm{~m}$ of water depth.

\subsection{Finite differences method}

Although the above equations have been linearized, they are still partial differential equations. Thereby, by using the finite differences method they are transformed in a set of ordinary differential equations which is achieved by discretizing the equations (4) and (5).

According this procedure the following set of ODE is obtained, and presented in the state space representation (Rijo, et al., 1992):

$$
\begin{aligned}
\frac{\partial h_{1}}{\partial t} & =-\frac{1}{B_{0_{1}}} \frac{q_{2}-q_{1}}{\Delta x} \\
\frac{\partial h_{n}}{\partial t} & =-\frac{1}{B_{0_{n}}} \frac{q_{n}-q_{n-1}}{\Delta x}
\end{aligned}
$$

There is no need of any equations for $q_{l}$ or $q_{n}$ as they are external boundary conditions.

It is known that this operation leads to a set of $2 n-2$ equations, if $n$ is the number of nodes used in discretizing.

\section{CONTROL STRATEGY AND SETTINGS}

\subsection{Control Strategy}

In the present paper a traditional PI controller will be adjusted to the specifics of the water canal prototype in study. The parameter settings for the proportional and integral gains was derived by comparing the frequency response of the state space model, which relies on the linearized Saint-Venant equations, with a simple model with the assumption of each pool as a reservoir (reservoir model)

So, assuming each pool as a reservoir, water depth and flow are related by

$$
\frac{d}{d t} H=\frac{1}{A_{\text {Sup }}} \Delta Q
$$

where $H$ is the water depth, $Q$ the discharge and $A_{\text {Sup }}$ is the superficial area of the pool.

Applying the Laplace transform on eq. (13) leads to:

$$
H=\frac{1}{s A_{\text {Sup }}}\left(Q_{\text {in }}-Q_{\text {out }}\right)
$$

where $s$ is the Laplace variable.

$Q_{\text {in }}$ and $Q_{\text {out }}$ can be thought as the upstream and downstream discharges for the considered pool of the canal for better understanding of this procedure.

The PI-control action can be written,

$$
u(s)=\left(K_{P}+\frac{K_{I}}{s}\right) e(s)
$$

Considering the water depth as the controlled variable and the discharges as control actions, equation (15) yields

$$
Q_{\text {out }}=\left(K_{P}+\frac{K_{I}}{s}\right)\left(H_{r e f}-H\right)
$$

for upstream water depth control.

Combining equations (14) and (16) it follows:

$$
\begin{gathered}
H=\frac{\frac{K_{P}}{A_{\text {Sup }}} s+\frac{K_{I}}{A_{\text {Sup }}}}{s^{2}+\frac{K_{P}}{A_{\text {Sup }}} s+\frac{K_{I}}{A_{\text {Sup }}}} H_{r e f}- \\
-\frac{s}{s^{2}+\frac{K_{P}}{A_{\text {Sup }}} s+\frac{K_{I}}{A_{\text {Sup }}}} Q_{\text {in }}
\end{gathered}
$$

assuming already that both gains are negative.

Equation (17) is a second order transfer function which has the standard format:

$$
G(s)=\frac{\omega_{n}^{2}}{s^{2}+2 \omega_{n} \xi s+\omega_{n}^{2}}
$$

where $\varpi_{n}$ is the natural frequency of the system and $\xi$ the damping ratio.

Combining equations (17) and (18) leads to quite simple expressions for $K_{P}$ and $K_{I}$.

$$
\begin{gathered}
K_{P}=-2 \omega_{n} \xi A_{\text {Sup }} \\
K_{I}=-\omega_{n}^{2} A_{\text {Sup }}
\end{gathered}
$$

The value of $\omega_{n}$ adopted will depend of the Bode response of both systems, i.e., the selected value for calculations has to correspond to a frequency where both systems have similar behaviors.

The frequency value will be related with the lowest resonance frequency of the system by

$$
\omega_{n}=\omega_{r} / n
$$

where $n$ is an integer number that is chosen according to the condition mentioned above. In all calculations, $\xi$ will be assumed as 1 , as it is the value that better fits, experimentally, to this canal.

\subsection{Parameter Settings}

The Bode plot for the first pool of the canal (Fig. 8) shows clearly that both models respond approximately until a certain value of frequency any controller that will be designed must take that fact in consideration.

All these plots were computed for the first pool of the canal with all its specific geometric characteristics and assuming the transfer function $\partial H_{n} / \partial Q_{n}$.

It is possible to identify the value of the frequency of the lowest resonance peak as well as the value where the differences of both models become significant (Figure 9).

It can be seen by analysing the Bode plot above that any value of $n$ (equation (21) has to be greater or equal than 4 . So, the values of $n$ chosen and the correspondent proportional / integral gains calculated 
by equations (19) and (20) are shown in Table 1.

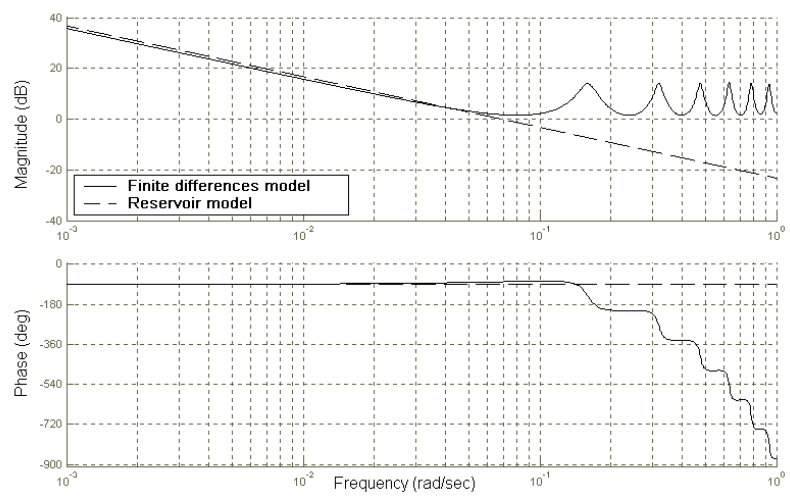

Fig. 8 - Bode Diagram for the canal $1^{\text {st }}$-pool (both reservoir and finite differences models)

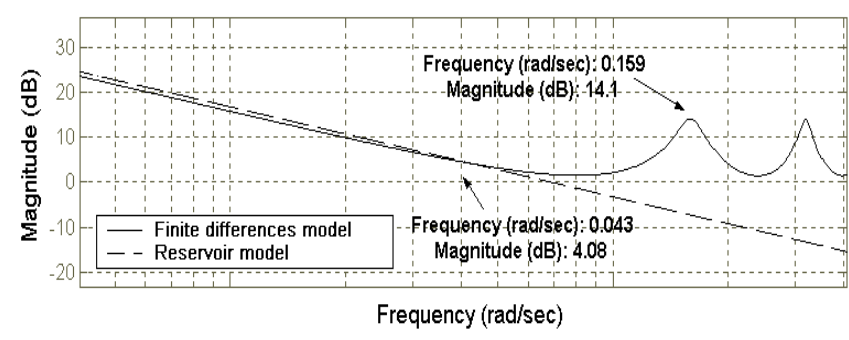

Fig. 9 - Bode Magnitude Diagram showing the lowest resonance frequency

$\underline{\text { Table } 1 \text { Controller-Gain settings }}$

\begin{tabular}{cccc} 
Controller & $\boldsymbol{n}$ & $\boldsymbol{K}_{\boldsymbol{P}}$ & $\boldsymbol{K}_{\boldsymbol{I}}$ \\
\hline$A$ & 4 & $-1,2$ & $-0,024$ \\
$B$ & 6 & $-0,8$ & $-0,010$ \\
$C$ & 8 & $-0,6$ & $-0,006$ \\
\hline
\end{tabular}

\section{CONTROLLER IMPLEMENTATION}

\subsection{Hardware constraints}

The physical implementation of the above designed PI-controller has to take into account two main hardware constraints: stop-time between consecutive control actions and hysteresis effects (Almeida, et al., 2002). Fig. 10 shows how these two effects have been managed.

In relation to the hysteresis effect, the controller only leads to an actuation when the error reference/output is greater than hysteresis (or minus hysteresis). Basically it is defined an allowable error range where no action will be taken. It was experimentally tested that this procedure stabilized the system. Without this constraint the gate control action was unstable, opening and closing continuously.

For the water canal prototype under study, with valve opening ranges from 0 to $100 \%$ and gates from 0 to $800 \mathrm{~mm}$, it was measured an hysteresis of $0,5 \%$ and $5 \mathrm{~mm}$ respectively. This means, for example, for gate actuation, control actions smaller than $5 \mathrm{~mm}$, are filtered out (no output signal is sent).

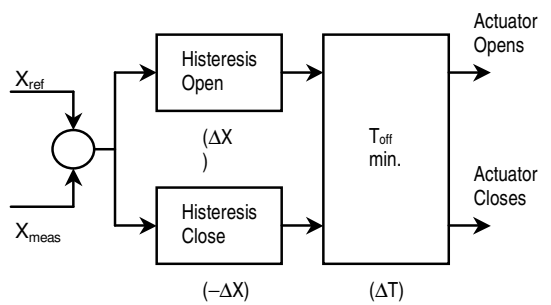

Fig. 10 - Management of hysteresis and stop-time at actuators

\subsection{Upstream/ Downstream water depth control}

To control the upstream or downstream water depth near the gate it was developed a strategy, which is shown in Fig. 11.

In the developed strategy the calculated water depth error is first passed through a Dead-Band filter. The output of the Dead-Band filter will generate a signal if the error value is smaller than the band width. Additionally to the Dead-Band filter a second-order filter is in place to eliminate measurement noise.

The PI-controller supplies an estimated flow which multiplied by the $\mathrm{Kx}$ parameter (gate parameter that relates gate opening with correspondent flow) gives the new gate position. In order to ensure that only allowable signals, in magnitude, are generated, a saturation block filters the calculated gate position. Finally the servo-motor position controller (SV) applies the signal to the gate.

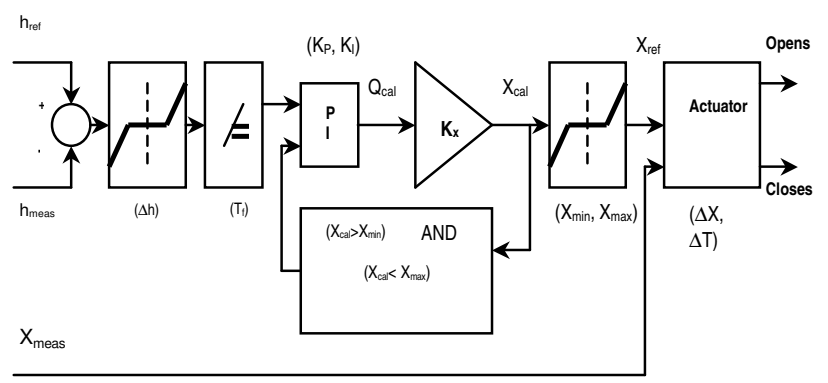

Fig. 11 - Upstream/Downstream control

\section{EXPERIMENTAL RESULTS}

The main menu of the developed SCADA application is presented in Fig. 12. This synoptic is a complete representation of the canal prototype reflecting the states of all inputs and outputs managed in this facility.

All the tests that will be presented in this section have been performed on the canal first pool and first gate. Controller $\mathrm{C}$ had delivered the best results and they will be present in the following figures.

As mentioned before, the project nominal features of the experimental canal are a $0,090 \mathrm{~m}^{3} / \mathrm{s}$ flow and a $0,070 \mathrm{~m}$ uniform water depth. So, the set-point in water depth used in all testing was precisely $0,700 \mathrm{~m}$, having the flow varying from $0,020 \mathrm{~m}^{3} / \mathrm{s}$ to a maximum of $0,080 \mathrm{~m}^{3} / \mathrm{s}$ and the other way down. 
The maximum project flow was not achieved because a $0,090 \mathrm{~m}^{3} / \mathrm{s}$ value was found to be quite severe for the installed equipments. Nevertheless, there are three distinct scenarios: the flow was modified in steps of $0,010 \mathrm{~m}^{3} / \mathrm{s}$, correspondent to $11 \%$ of the canal global capacity, $0,020 \mathrm{~m}^{3} / \mathrm{s}(22 \%)$ and $0,030 \mathrm{~m}^{3} / \mathrm{s}(33 \%)$.

In spite of testing had not included any off-take operations, the results can easily be thought as so, because the final selected controller will prove its capability of bringing the water level back to the desired set-point, independently of the disturbance epicenter, flow or off-take opening. In other words, the controller will be capable of responding properly to all upstream disturbances.

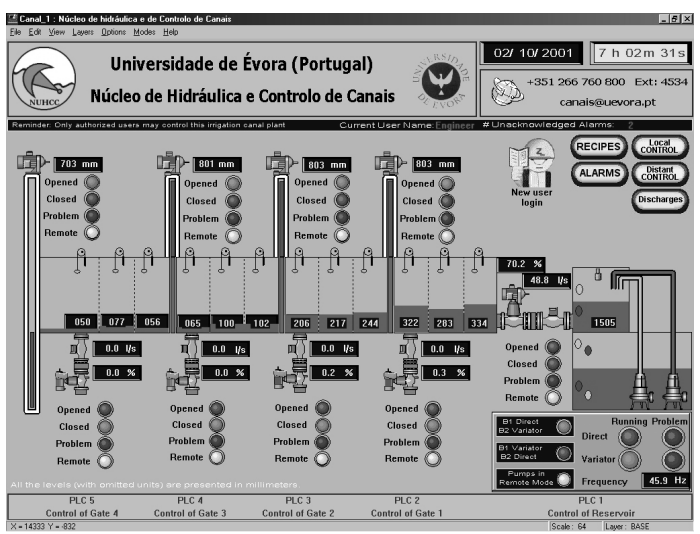

Fig. 12 - SCADA annlication (main menu)

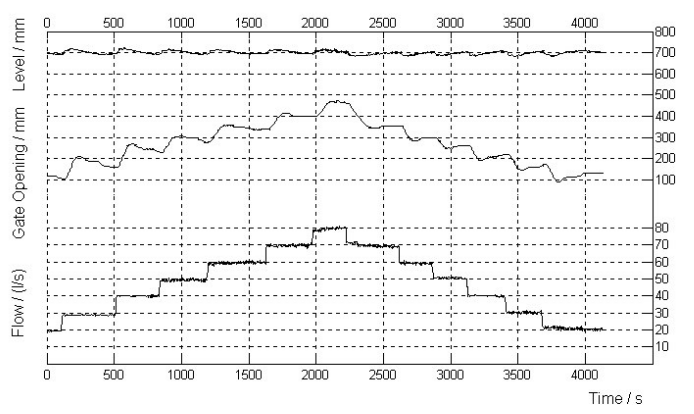

Fig. 13 - Controller C performance with discharge step disturbances of $0,010 \mathrm{~m}^{3} / \mathrm{s}$

\section{CONCLUSIONS}

The main purpose of this paper was to study upstream/downstream water depth control in an automatic water canal prototype and, at the same time, providing this automatic facility with the basic tools to be managed, in the future, with more robust and sophisticated control approaches. Specifically regarding the results supplied by Controller-C it can be concluded that the PI strategy applied to such big delay system had shown good results.

Regarding the specifics of this canal, it can be pointed out that the controller performance could be improved by decreasing the hysteresis factor, but this risks the stability of the overall system. The $\mathrm{Kx}$ parameter (s. 5.2) is a canal/gate shape characteristic and its value must remain independently of the chosen control strategy. The Time-Off parameters should also remain with the same values in order to preserve the plant integrity. All other parameters can be easily changed according to other control strategies to be implemented in the future.

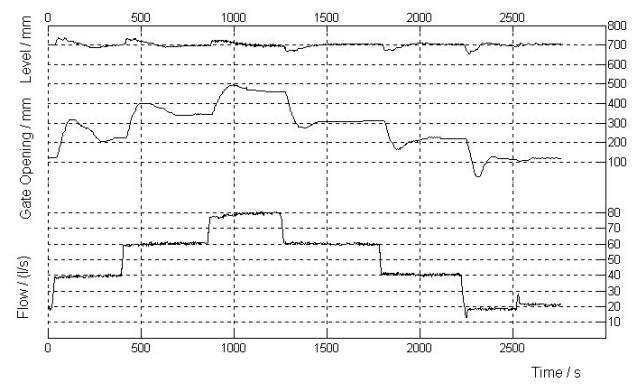

Fig. 14 - Controller $\mathrm{C}$ performance with discharge step disturbances of $0,020 \mathrm{~m}^{3} / \mathrm{s}$



Fig. 15 - Controller $\mathrm{C}$ performance with discharge step disturbances of $0,030 \mathrm{~m}^{3} / \mathrm{s}$

\section{REFERENCES}

Almeida, M., Figueiredo, J., Rijo, M. (2002). SCADA Configuration and Control Modes Implementation on an Experimental Water Supply Canal. Proc. $10^{\text {th }}$ IEEE Mediterranean Conf. On Control and Automation - MED2002, Lisbon, 2002

Baume, J.P., Malaterre, P., Sau, J. (1999). Tuning of PI Controllers for an Irrigation Canal using Optimization Tools. Proc. USCID Ws Modernization of Irrigation Water Delivery Systems, Phoenix, 1999, p. 483-500

Rijo, M. (1999). SCADA of an Upstream Controlled Irrigation Canal System. Proc. USCID Workshop - Modernization of Irrigation Water Delivery Systems, Phoenix, 1999, p. 123-136

Rijo, M., Almeida, A, Pereira, L. (1992). Math. Modelling and Field Study of Unsteady Flow in an Irrigation Canal System. Proc. Hydrosoft'92, Valencia, 1992, Computation Mechanics Publ. \& Elsevier Applied Science, London, p. 339-349,

Schuurmans, J. (1997). Control of Water Levels In Open Channels, Ph.D thesis, 223 p., ISBN 909010995-1 Delft, Netherlands, 1997 